Bio - grafía. Escritos sobre la Biología y su Enseñanza. ISSN 2027-1034

Edición Extraordinaria. p.p. 926 - 932

Memorias del IX Encuentro Nacional de Experiencias en Enseñanza de la Biología y la Educación Ambiental. IV Congreso Nacional de Investigación en Enseñanza de la Biología.

\title{
JUGUEMOS A RESPIRAR. COMO ENTENDER EL CONCEPTO DE RESPIRACIÓN DESDE EL JUEGO DE PONCHADOS EN LA CLASE DE EDUCACIÓN FÍSICA.
}

\section{LET'S PLAY BREATHING. HOW TO UNDERSTAND THE CONCEPT OF BREATHING FROM THE GAME OF PONCHADOS IN THE CLASS OF PHYSICAL EDUCATION.}

\author{
Mónica Patricia Melo Herrera ${ }^{1}$
}

\section{RESUMEN}

El aprendizaje de conceptos de ciencias desde otras áreas de conocimiento puede contribuir al fortalecimiento de la transversalidad de los saberes y la innovación en el aula. El presente trabajo muestra los resultados obtenidos del desarrollo del tema de la respiración desde actividades prácticas como el juego de ponchados durante las clases de educación física. Para ello se empleó el método experimental participativo con estudiantes de grado octavo de colegio IEDIT Rodrigo de Triana. Los resultados mostraron que los estudiantes comprendieron con mayor facilidad el proceso de respiración con actividades corporales que le exigían cambiar sus propios ritmos cardiacos, además de ello, logro explicar aspectos claves de este proceso humano y tomo conciencia de la importancia de pensar su propia respiración.

\section{SUMMARY}

The learning of science concepts from other areas of knowledge can contribute to strengthening the transversality of knowledge and innovation in the classroom. The present work shows the results obtained from the development of the subject of breathing from practical activities like the game of strikeouts during physical education classes. For this purpose, the participatory experimental method was used with eighth grade students from the IEDIT Rodrigo de Triana school. The results showed that the student more easily understood the breathing process with bodily activities that required him to change his own heart rhythms, managed to explain key aspects of this human process and became aware of the importance of thinking his own breathing.

\section{INTRODUCCIÓN}

Los procesos de enseñanza y aprendizaje en la escuela vienen cambiando constantemente, sin embargo, los métodos empleados para tal fin, en algunos casos, siguen siendo anquilosados y persiste en el ambiente escolar que el estudiante es una hoja en blanco que se debe diligenciar.

\footnotetext{
${ }^{1}$ Docente Secretaria de Educación de Bogotá, Magister en Didáctica de las Ciencias, Trabajo realizado en la institución educativa IEDIT Rodrigo de Triana en la cuidad de Bogotá.
} 


\title{
Bio - grafía. Escritos sobre la Biología y su Enseñanza. ISSN 2027-1034
}

\section{Edición Extraordinaria. p.p. 926 - 932}

\author{
Memorias del IX Encuentro Nacional de Experiencias en Enseñanza de la Biología y la \\ Educación Ambiental. IV Congreso Nacional de Investigación en Enseñanza de la \\ Biología.
}

Este hecho ha puesto de manifiesto la necesidad de cambiar las prácticas educativas, innovar tanto en los currículos como en los modelos didácticos y entender al educando como un ser lleno de saberes que pueden ser articulados a nuevos conocimientos y de esta manera, promover un espíritu crítico y reflexivo de los elementos que circundan su vida. De ahí se desarrolló una propuesta didáctica a partir del juego, que pretendía mostrarle al mismo estudiante que los saberes no son exclusivos de un área del conocimiento y que muchos de ellos están latentes en la vida cotidiana de cada uno, como lo es el andar, el comer, el respirar.

Jugar va más allá de una simple actividad donde se gasta energía y se pasa el tiempo. A través de la historia el juego ha desempeñado un papel importante en la construcción del sujeto, la cultura y la sociedad, permitiendo crear diversas relaciones con objetos, situaciones, personas; de esta manera se potencia el desarrollo cognitivo, la resolución de problemas y da origen a nuevos conocimientos. El historiador Johan Huizinga (2000) manifestó que el juego fue más viejo que la misma cultura; que los seres humanos son lúdicos por naturaleza, y que ello permitía considerar al juego como un elemento de construcción tanto del ser humano como de su entorno.

Desde el aprendizaje se entiende al juego como un proceso de asimilación, al mismo tiempo que permite dar significado a las cosas y situaciones a partir de las relaciones que el sujeto establece con ellas; durante este proceso se crean confrontaciones internas con lo que se conoce y lo que es nuevo (Piaget, 1994). Esta actividad en el desarrollo del ser humano ha permitido entender cómo aprende y, cuáles son sus transformaciones y las de su entorno.

Ahora bien, si el juego permite desarrollar procesos de aprendizaje en la escuela ¿por qué ha sido subvalorado como recurso didáctico? A partir de este cuestionamiento, se realizó una aplicación de aula, basada en el método experimental participativo de Paulo Freire, el cual se basa en la participación y concientización como resultado de la construcción del pensamiento, donde las personas inmersas, en este caso los estudiantes, hacen parte de la elaboración del conocimiento y no se limitan sólo a recibir información; de esta manera se toma conciencia de lo que se está haciendo y de la importancia que trae consigo; de ahí que el mencionaba que "la reflexión crítica sobre la práctica se torna una exigencia de la relación teoría/práctica sin la cual la teoría puede convertirse en palabrería y la práctica en activismo" (1974).

\section{METODOLOGÍA}

Para abordar el tema de la respiración desde las clases de educación física con los estudiantes de grado octavo se diseñó una unidad didáctica en la cual se articularon los conceptos que los estudiantes vieron en las clases de ciencias y que debían identificar la actividad que se proponía, en este caso, los ponchados, un juego de la calle tradicional en Colombia.

La respiración en términos generales es entendida como el proceso fisiológico vital para los organismos aeróbicos (Pelizzari \& Tovaglieri, 2007) y de cómo el ser humano obtiene 


\title{
Bio - grafía. Escritos sobre la Biología y su Enseñanza. ISSN 2027-1034
}

\section{Edición Extraordinaria. p.p. 926 - 932}

\author{
Memorias del IX Encuentro Nacional de Experiencias en Enseñanza de la Biología y la \\ Educación Ambiental. IV Congreso Nacional de Investigación en Enseñanza de la \\ Biología.
}

la energía y los nutrientes de la alimentación de una manera adecuada. Visto desde una mirada menos coloquial, se puede decir que la función primordial de la respiración es "proporcionar oxígeno a la sangre arterial y eliminar anhídrido de la sangre venosa. Para que esto se cumpla, se requiere la actuación integrada de diversos eslabones fisiológicos" (Escola, 1989. P. 11). Estos eslabones fisiológicos garantizan el correcto funcionamiento del aparato respiratorio que va más alla de una ventilación ya que este proceso permite, además de renovar el gas que alcanzan los alveólos y del intercambio gaseoso con la penetración del oxígeno y la salida del anhídrico carbónico, permiten que el oxígeno llegue a la sangre arterial para ser tranportada a los tejidos por medio de la circulación y de una correcta función cardiaca.

Este trabajo se orientó para que el estudiante adquiriera una mejora propioceptiva, entendida esta como la capacidad que tiene el cuerpo de sentirse y entenderse (Chance, 2013); en este caso se buscaba que los jóvenes logran comprender con mayor detenimiento el proceso de respiración, que reconocieran las partes del cuerpo que intervienen en esta actividad, que adquirieran conciencia de la importancia de respirar adecuadamente y que identificaran otras capacidades físicas que intervienen durante el desarrollo de las clases.

Para el diseño de la unidad didáctica se tuvo como soporte pedagógico el método experimental participativo propuesto por Paulo Freire ya que todos los estudiantes debían participar y describir sus propias experiencias y relacionar las experiencias de los otros miembros del grupo a través de la comparación. Para abordar el tema propuesto se tiene como de partida los conocimientos adquiridos en las clases de ciencias sobre el tema de respiración mientras que el docente se convierte en un asesor, facilitador, esclarecedor de dudas y el que propicia en los estudiantes una reflexión de lo realizado.

En un primer momento los estudiantes se disponen como todas las clases de educación física a realizar las actividades propias del área, con la indumentaria adecuada y con la preparación previa al tema principal, en este caso, el calentamiento. Durante este se les hacían preguntas de los conceptos que ya se habían desarrollado en otras sesiones y en qué momento los identifican en la sesión.

Posterior a ello debían mencionar los órganos que componen el sistema respiratorio. Se les solicitó que cada uno los identificara en su cuerpo y recordaran que función cumplía cada uno. Se les indicó que se iba a realizar el juego de ponchados con el fin que identificaran cómo se desarrolla el proceso de respiración en su cuerpo, que analizaran los cambios que se producían con la actividad y que pensaran qué otras habilidades se involucran en el juego y que intervienen en la respiración.

Como es un trabajo participativo, en su gran mayoría respondían las preguntas iniciales; mencionaron partes del sistema respiratorio como los senos paranasales, nariz, tráquea, faringe bronquios, pulmones entre otros. Se les pidió que cada uno los señalara en su cuerpo a medida que ellos mismos los iban mencionando y que recordaran que función cumplía cada uno. 
Bio - grafía. Escritos sobre la Biología y su Enseñanza. ISSN 2027-1034

Edición Extraordinaria. p.p. 926 - 932

Memorias del IX Encuentro Nacional de Experiencias en Enseñanza de la Biología y la

Educación Ambiental. IV Congreso Nacional de Investigación en Enseñanza de la Biología.

En el momento de la actividad central, en este caso el juego, los estudiantes ya habían manifestado cambios en su cuerpo y respiración con el calentamiento que consistía en caminar en zigzag en medio de unos platillos dispuestos en el piso del patio de un extremo hasta el otro de la cancha principal y luego regresar haciendo saltos laterales. Al manifestar estos cambios, se les indicó que pensaran qué transformaciones específicas había tenido en el cuerpo y que posterior a ello debían describirlo de manera oral al finalizar la clase y de manera escrita para el cuaderno de la materia (Ver imagen 1).

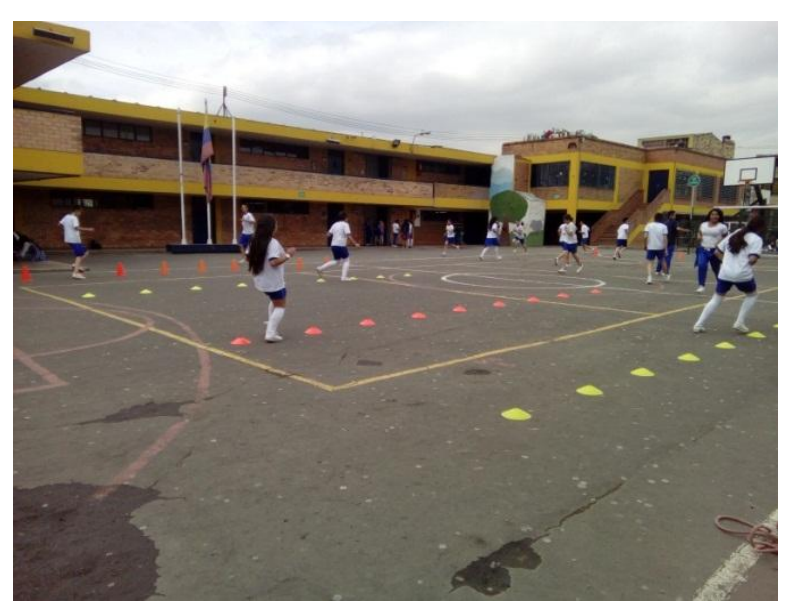

Imagen 1. Patio central del colegio donde los estudiantes realizan el calentamiento.

Luego de ello se realizó el juego de los ponchados que consiste en lanzar una pelota al aire, mencionar el nombre de un integrante del grupo que será el encargado de lanzar la pelota al cuerpo de algún compañero y si fuese efectivo el lanzamiento gritar "ponchado". Durante la actividad no se podían salir de los límites del patio central. Para no ser alcanzado por la pelota se debe correr y tocar unas bases establecidas como comodines, en este caso las astas, el árbol pintado en la pared y la salida/legada. Al final se debe llegar al mismo punto de salida sin ser tocado por la pelota. El tiempo de duración de esta actividad fue de 30 minutos (Ver imagen 2). 
Bio - grafía. Escritos sobre la Biología y su Enseñanza. ISSN 2027-1034

Edición Extraordinaria. p.p. 926 - 932

Memorias del IX Encuentro Nacional de Experiencias en Enseñanza de la Biología y la Educación Ambiental. IV Congreso Nacional de Investigación en Enseñanza de la Biología.

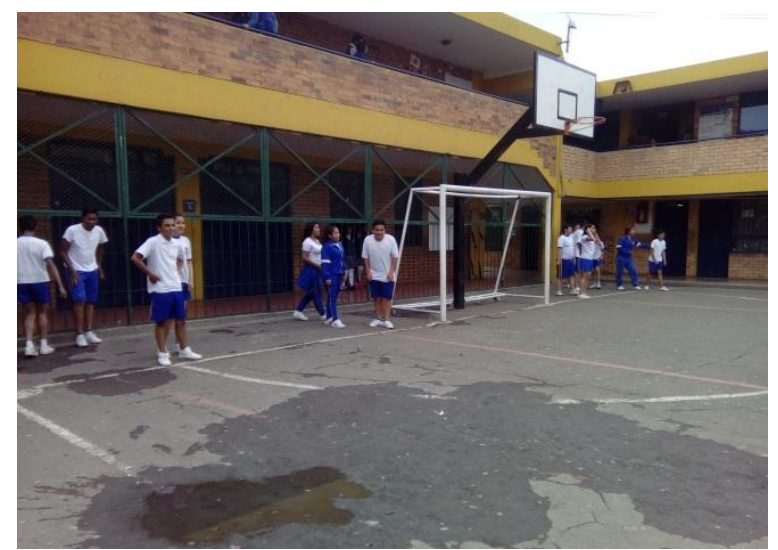

Imagen 2. Punto de salida del juego "Ios ponchados"

Al finalizar el juego se procedió a realizar la vuelta a la calma, momento en el cual el cuerpo va disminuyendo la intensidad del ejercicio de manera progresiva. En esta fase de la actividad se hace un conversatorio con todos los participantes, en este caso, todos los estudiantes de cada curso, donde se continúan haciendo las preguntas referentes al proceso de respiración; además de realizar la socialización de manera oral cada uno escribía los cambios que sufrió su cuerpo durante la actividad (Ver imagen 3).

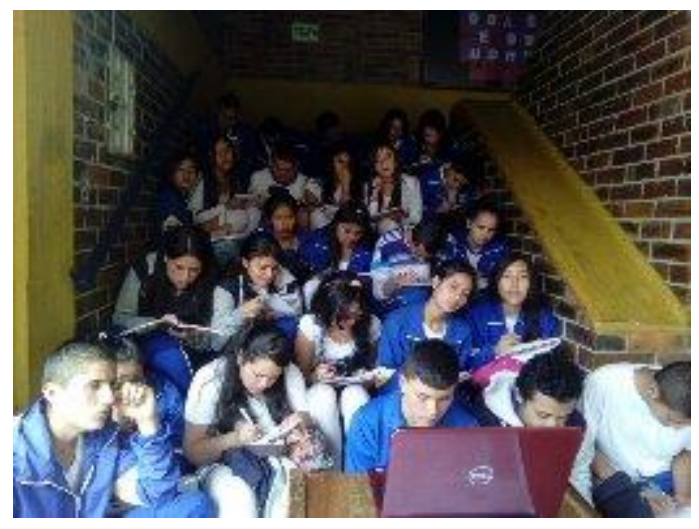

Imagen 3. Estudiantes registrando en el cuaderno los cambios que identificaron en su cuerpo durante la actividad.

\section{Resultados y discusión}

Al finalizar la jornada, los estudiantes dieron cuenta que comprendieron de mejor manera el concepto de respiración ya que identificaron claramente los órganos que intervienen en este proceso, reconocieron los cambios que experimentaron durante la actividad y lo 


\title{
Bio - grafía. Escritos sobre la Biología y su Enseñanza. ISSN 2027-1034
}

\section{Edición Extraordinaria. p.p. 926 - 932}

\author{
Memorias del IX Encuentro Nacional de Experiencias en Enseñanza de la Biología y la \\ Educación Ambiental. IV Congreso Nacional de Investigación en Enseñanza de la \\ Biología.
}

explicaron usando un vocabulario más cotidiano, por ende, más entendible para ellos. Manifestaron que era más agradable hacer trabajos donde implicaba un movimiento corporal y aún más si estaban jugando ya que podían reírse, sentir más emociones, apoyar a otros compañeros entre otros. De hecho, el juego se establece como "un aliado de las actividades en el aula y al aire libre por su carácter motivante y divertido... al mismo tiempo que se aprende se estimulan las capacidades del pensamiento, la creatividad y el espíritu investigativo" (Melo H y Hernández B, 2014)

Entre esas respuestas mencionaron que sentían como el aire llegaba a su nariz y pasaba por la faringe, laringe hasta llegar a los pulmones y cómo este lograba darles la energía que necesitaban para poder soportar el ejercicio.

Mencionaron cambios en la temperatura debido al aumento de velocidad de la circulación y entendieron que este aumento se debía a que el cuerpo requería mayor capacidad de oxígeno, que no eran solo los pulmones los involucrados en la respiración.

Explicaron que el corazón comenzaba a latir más fuerte porque todas las partes del cuerpo requerían oxígeno, energía, y que la sangre arterial permitía este proceso. Mencionaron que si había deficiencia de oxígeno se podía tener alteraciones cardiacas y que por ello era importante la respiración.

Se les preguntó que si faltaba alguno de los órganos que ellos mencionaron que pasaría. En su mayoría respondieron que no se podría hacer una actividad como la que hicieron; algunos mencionaron que tendrían que tener aparatos artificiales para respirar y otros mencionaron que no se podía vivir sin respirar.

Al preguntarles si identificaron otros conceptos vistos anteriormente durante el juego de ponchados, y que fueran necesarios para el proceso de respiración, mencionaron que encontraron a resistencia aeróbica; indicaron que ella era la que les permitía aguantar el tiempo requerido para participar durante todo el juego; también mencionaron la fuerza que necesitaron para lanzar la pelota y que al ejecutar este movimiento respiraban con más profundidad, de esta manera demostraron que ellos mismos comprendieron que existe una relación de los contenidos anteriormente vistos y de otras áreas de conocimiento que ven en la escuela.

El estudiante al identificar que existe una transversalidad en los contenidos de distintas materias, comprendió que es importante tener presentes los temas que ha visto, ya que en algún momento durante sus actividades académicas le pueden servir. En este sentido Yus (1996) citado en Paredes y Ávila (2008) plantea que los temas transversales son:

"conductores de la actividad educativa que, sin estar ligados a ninguna materia en particular, se puede considerar que son comunes a todas, por formar parte esencial de la educación y la socialización de las personas, y de interés por sus efectos potenciales para la sociedad y la supervivencia del individuo".

A partir de esta práctica lúdica donde se desarrolló el tema de la respiración se pudo mostrar que el estudio de las ciencias en la escuela debe dejar de ser visto como una tarea exclusiva del área de conocimiento y en donde el docente es el que domina el 
Bio - grafía. Escritos sobre la Biología y su Enseñanza. ISSN 2027-1034

Edición Extraordinaria. p.p. 926 - 932

Memorias del IX Encuentro Nacional de Experiencias en Enseñanza de la Biología y la Educación Ambiental. IV Congreso Nacional de Investigación en Enseñanza de la Biología.

conocimiento y el estudiante se limita a escuchar y seguir instrucciones. En este sentido, los planteamientos didácticos que se describen han servido para que el estudiante desde una práctica cotidiana como el jugar ponchados logre identificar que los aprendizajes no son aislados de los componentes de la vida, y que, al contrario, se debe tener conciencia de ello; y esa toma de conciencia de lo aprendido es el objetivo. Freile citado por Torres considera que la concientización es algo que va más allá...es algo que implica analizar. Es una manera de leer el mundo rigurosamente (s.f).

\section{BIBLIOGRAFÍA}

Chance, J. (2013). La técnica Alexander: las posturas del bienestar. Barcelona: Amat.

Escola Balaguero, F. (1989). Educación de la respiración. Pedagotecnia para el rendimiento físico y la fonación. Barcelona: Inde.

Freire, P. (1974). En concientización, teoría y práctica de la liberación. Sao Paulo: Bs.

Huizinga, J. (2000). Homo Ludens. (E. Imaz, Trad.) España: Alianza/Emecé.

Melo H, M., \& Hernández B, R. (2014). El juego y sus posibilidades en la enseñanza de las ciencias naturales. Innovación educativa, 41-63.

Paredes, I., \& Ávila, M. (2008). La transversalidad curricular como eje conductor para la paz. Lauris, 281-301.

Pelizzari, U., \& Tovaglieri, S. (2007). Curso de Apnea. Badalona: Paidotribo.

Piaget, J. (1994). La Formación del Símbolo en el Niño. (Gutierrez, Trad.) Colombia: Fondo de desarrollo Económico.

Torres, C. (s.f). Estudios Freireanos. Argentina: Coquena Grupo. 